\title{
THE USNO (FLAGSTAFF STATION) CCD TRANSIT TELESCOPE AND STAR POSITIONS MEASURED FROM EXTRAGALACTIC SOURCES
}

\author{
RONALD C. STONE AND DAVID G. MONET \\ U. S. Naval Observatory, Flagstaff Station \\ P.O. Box 1149 \\ Flagstaff, $A Z$ 86002
}

\begin{abstract}
The USNO (Flagstaff Station) is in the process of modernizing its 8-inch transit telescope. The upgraded instrument will use a CCD detector to measure the positions of both bright and faint stars directly with respect to extragalactic sources. Moreover, it will be able to observe large numbers of stars very rapidly, and improvements in accuracy are expected with further upgrades (e.g. CCD circle scanners, an interferometric telescope monitoring system, networks of environmental sensors). Many research opportunities related to coordinate systems exist for this telescope.
\end{abstract}

\section{Star Positions from an Extragalactic Reference Frame}

The U.S. Naval Observatory at Flagstaff is converting its 8-inch transit circle into a CCD scanning telescope that will be able to measure star positions differentially from extragalactic sources (QSO's, compact galaxies, and BL Lac objects) and radio stars with good radio positions. These objects can define an inertial reference frame.

The observing will be done in scan mode. The telescope will be set at a given declination and will scan the sky continuously by clocking the charge image of the sky across the CCD. The field of view of the current CCD chip is $20^{\prime}$ by $20^{\prime}$, and the telescope has the capacity to determine the centers of all the images in this area that are unblended and within the usable range of magnitudes. This is often more than 50 star images per field, and star positions should be measurable at a rate of 2000 images per hour. The limiting magnitude of the telescope is $\mathrm{V} \approx 17.5 \mathrm{mag}$, and with a combination of short exposures and the use of screens for magnitude attenuation, stars as bright as $\mathrm{V} \approx 5.0$ mag should also be observable. Magnitudes with accuracies under \pm 0.05 mag can also be determined.

Koo, Kron, and Cudworth (1986) give counts of QSO's as a function of apparent magnitude. By using their statistics, the 8-inch TT is expected to observe 10 anonymous QSO's in each hour of scanning, except of course at low Galactic latitudes. Unfortunately, very few of these objects have been identified and even less have well determined radio positions. According to de Vegt (1986), 
about 233 extragalactic objects have good radio positions, of which the 8-inch should be able to observe 77 (or $33 \%$ ) of them. All reductions will be made differentially, thereby minimizing many of the sources of systematic error (e.g. refraction, flexure, and clock errors).

\section{The Refurishment of the 8-inch Telescope}

The current 8-inch CCD detector is a thinned, back-illuminated, buried channel, Texas Instruments chip which has $800 \times 800$ pixels, a pixel size of 15 microns, and is cooled with liquid air. The current filter is the Hubble telescope W606 "Wide V" which has a passband of $4800-7200 \AA$. In spite of the broad passband, the expected color equation (using the simulations discussed by Stone 1984) is no larger than that of the traditional V-passband. All processing is controlled with a DEC micro-VAX II and associated periperals.

The detector system has no moving parts. The CCD is mounted in the focal plane of the objective, and the tracking of stars is achieved by shifting the charge (column by column) along the chip at the diurnal rate. Once a column is shifted into the serial register, it is read and then transferred to a data file for further processing. Thus, star images can be scanned and read in a continuous process which can extend for many hours. Instrumental motions can be monitored in declination with readings of the Heidenhahn circle and in azimuth and level with four Hewlett Packard laser interferometric systems. The monitoring will be done in real-time. The old mechanical circle scanners of the telescope have been replaced with CCD scanners which are very accurate and stable. There are six scanners altogether, and the internal accuracy of a single scan made by one of the units is around \pm 0.01 arcsec.

Even if the telescope is very stable, the apparent zenith distances of stars can be altered with changes in the value of refraction occurring in the course of the scanning. This can be controlled by applying a correction for refraction to the observations as determined from the changing ambient observing conditions. The refraction appropriate for the passband is determined with the numerical technique discussed by Stone (1984). Environmental monitoring of temperature, pressure, and dew point is done at high accuracy and with triple redundancy. Accurate time is needed for the determination of right ascensions. The clock system consists of two Hewlett Packard high precision Cesium beam clocks (which are monitored with LORAN-C receivers) and converters for sidereal time.

\section{References}

de Vegt, C. 1986, in IAU Sympos. No. 109, Astrometric Techniques, (Reidel, Dordrecht), p173.

Koo, D.C., Kron, R.G., and Cudworth, K.M. 1986, P.A.S.P. 98, 285.

Stone, R.C. 1984, Astron. Astrophys. 138, 275. 